\title{
Schlusswort: Wertschätzung für und in Kitas geht uns alle an
}

Viel Erfolg für alle Kita-Leitungen An dieser Stelle möchten wir Ihnen als Kita-Leitung für die Führung Ihrer Mitarbeiter/-innen in Krippe, Kindergarten und Hort viel Erfolg wünschen. Wir hoffen, dass dieses essential das „Leben und Arbeiten" in Kitas tatsächlich entscheidend verbessern hilft - für Sie als Kita-Leitung, für Ihre Mitarbeiter/-innen und natürlich für die in Ihrer Einrichtung betreuten Kinder und deren Eltern.

Mehr Wertschätzung bitte! Außerdem hoffen wir, Ihnen die besondere Bedeutung von Wertschätzung im Rahmen Ihrer Tätigkeit als Kita-Leitung nähergebracht zu haben. Denn Wertschätzung spielt nicht nur in der Kita, sondern auch in unserer gesamten Gesellschaft eine bedeutsame Rolle. Wertschätzung ist ein Grundbedürfnis, das jeder Mensch hat. Zudem ist Wertschätzung ein zentraler Pfeiler von Bildung und Entwicklung über die gesamte Lebensspanne. Wir brauchen ein Mehr an Wertschätzung in unserer Gesellschaft. Denn nur wer wertgeschätzt wird, kann auch lernen und sich entwickeln - das gilt nicht nur für Kinder, sondern auch für Erwachsene: Wertschätzung ist wichtig für Groß und Klein!

Wertschätzung für Groß und Klein - von Anfang an, ein Leben lang! Als Kita-Leitung haben Sie jetzt die Chance, die gesellschaftlich so wichtige Wertschätzung für und in Ihrer Einrichtung (sei es Kindergarten, Krippe oder Hort) mitzugestalten und voranzutreiben - für Sie selbst, für Ihre Mitarbeiter/-innen sowie für die Kinder und deren Eltern. Kitas sind eine (wenn nicht sogar die) zentrale Wiege der Wertschätzung unserer Gesellschaft im 21. Jahrhundert. Wertschätzende Bildung geht uns alle an - von Anfang an, ein Leben lang! 